\title{
Can Bohmian quantum information help us to understand consciousness?
}

Paavo Pylkkänen

Department of Philosophy, History, Culture and Art Studies and The Academy of Finland Center of Excellence in the Philosophy of the Social Sciences (TINT), P.O. Box 24, FI-00014 University of Helsinki, Finland.

Department of Cognitive Neuroscience and Philosophy, School of Biosciences, University of Skövde, P.O. Box 408, SE-541 28 Skövde, Sweden

E-mail: paavo.pylkkanen@helsinki.fi

\section{Introduction}

A key idea in the field of "quantum interaction" or "quantum cognition" is that certain principles and mathematical tools of quantum theory (such as quantum probability, entanglement, non-commutativity, non-Boolean logic and complementarity) provide a good way of modeling many significant cognitive phenomena (such as decision processes, ambiguous perception, meaning in natural languages, probability judgments, order effects and memory; see Wang et al. 2013). However, when we look at research in philosophy of mind during recent decades, it is clear that conscious experience has been the most important topic (see e.g. Chalmers ed. 2002; Lycan and Prinz eds. 2008). Even in the field of cognitive neuroscience consciousness has become a very important area of study (see e.g. Baars et al. eds. 2003). Might the principles and mathematical tools of quantum theory be also useful when trying to understand the character of conscious experience and its place in nature?

Note that many of the proposals in the area of "quantum mind" were not originally designed to deal specifically with the question of consciousness (i.e. with questions such as "What is it that makes a non-conscious mental state conscious?"). For example, Penrose's (1989) well-known proposal about orchestrated and objective quantum collapse (ORCH-OR) in neural microtubules was originally proposed to account for the presumably non-computational physical aspect of human intelligence, rather than conscious phenomenal experience per se. Yet, given that explaining the relationship of conscious experience to the physical domain is widely considered to be a truly hard problem (Chalmers 1996), it seems relevant and valid to consider 
whether our best theories of matter, such as quantum theory, might play a role in tackling it (cf. Atmanspacher 2015).

In this paper I will explore whether David Bohm's proposal about quantum theoretical active information, and the mind-matter scheme he developed on the basis of it, can help us to explain consciousness (Bohm and Hiley 1987, 1993; Bohm 1989, 1990; Pylkkänen 2007). Here it is important to acknowledge that other researchers in philosophy of mind and consciousness studies have also made use of the concept of information in their theories of mind and consciousness. For example, Dretske (1981) and Seligman and Barwise (1997) have explored the possibility that information in the sense of factual semantic contents (i.e. information as meaningful data that represents facts correctly or incorrectly) can be grounded in environmental information (i.e. information as mere correlation, e.g. the way tree rings carry information about age). For Dretske this was an important part of his attempts to give a naturalistic account of sensory experiences, qualia and consciousness. During recent years the notion of information has been used to explain consciousness most notably by David Chalmers (1996), as well as by Giulio Tononi and his co-workers (Tononi and Koch 2014; Oizumi, Albantakis and Tononi 2014). The strategy of this paper will be to first describe Bohm's mind-matter scheme, and then to briefly consider Chalmers' and Tononi et al.'s ideas in the light of this scheme.

\section{Bohm's mind-matter scheme based on quantum theoretical active information}

To understand the significance of Bohm's interpretation of quantum theory, which underlies his mind-matter scheme, it is necessary to briefly consider the development of physics in the $20^{\text {th }}$ century. When quantum theory was emerging, people were trying to make sense of puzzling features such as wave-particle duality and a little later, entanglement. In particular they were attempting to develop ontological models of quantum systems such as electrons. In the 1920s de Broglie came up with the idea of an electron being a particle guided by a pilot wave, while Schrödinger was trying to describe the electron as some kind of a physical field. These models had some difficulties, though in retrospect we can see that at least de Broglie's ideas could have been developed further (see Bacciagaluppi and Valentini 2009). What happened however was that the so-called "Copenhagen interpretation" won the day in the 1920s. There are actually many different versions of this interpretation, but it is 
typical of them that they emphasize epistemology - in the sense our ability to predict the results of measurement, rather than ontology - in the sense of a model of what quantum reality may be like also when we are not making measurements. As a result, physicists were not able to offer a new notion of objective physical reality, which philosophers could then use when discussing ontological issues, such as the mindmatter relation.

It is here that Bohm comes in. In the early 1950s, after discussions with Einstein in Princeton, he independently rediscovered de Broglie's theory and formulated it in a more coherent way, providing a first consistent realistic model of quantum systems (Bohm 1952). Bohm's interpretation was initially resisted, but is today more and more widely acknowledged as one of the key possible interpretations of quantum theory. Later on further ontological models were proposed, for example Everett's 1957 “many worlds" interpretation and Ghirardi, Rimini and Weber's 1986 objective collapse theory, and currently the nature of quantum reality is intensively debated within the philosophy of physics community (see Ney and Albert eds. 2013).

We do not know which ontological interpretation (if any) is correct, but each may reveal something significant about the nature of physical reality at a very fundamental level. One should note that there are by now also different versions of the Bohm theory. Much attention has in recent years been given to a minimalist version known as "Bohmian mechanics" (see Goldstein 2013; Dürr et al. 2013). Bohm himself developed since the mid-1970s, with Basil Hiley, a philosophically more radical version they called the "ontological interpretation", culminating in their 1993 book The Undivided Universe. While there has been a tendency in the Bohmian mechanics camp (see also Bacciacaluppi and Valentini 2009) to downplay the significance of Bohm and Hiley's approach, a more balanced examination suggests that both approaches have their value (see Holland 2011).

Let us now briefly consider the Bohm theory in some more detail. It assumes that every particle has a well-defined position and momentum and is accompanied by a field $\psi$ which satisfies the Schrödinger equation (eq. 1).

$$
\mathrm{i} \hbar \frac{\partial \psi}{\partial t}=-\frac{\hbar^{2}}{2 m} \nabla^{2} \psi+V(\boldsymbol{r}) \psi
$$

If we make a polar substitution (eq. 2) 
$\psi(\boldsymbol{r}, t)=R(\boldsymbol{r}, t) \exp [\mathrm{i} S(\boldsymbol{r}, t) / \hbar]$

and then separate out the real and imaginary parts, we find two equations, firstly (eq.

3)

$\frac{\partial S}{\partial t}+\frac{1}{2 m}(\nabla S)^{2}+V+Q=0$

where (eq. 4)

$$
Q=-\frac{\hbar^{2}}{2 m} \frac{\nabla^{2} R}{R}
$$

is known as the quantum potential.

The second equation (eq. 5)

$\frac{\partial P}{\partial t}+\nabla \cdot\left(P \frac{\nabla S}{m}\right)=0$

is a probability conservation equation. We also identify (eq. 6)

$p=\nabla S$

This is known as the guidance condition, from which the trajectory of the particle can be calculated. Figures 1 and 2 provide well-known visualizations for the two-slit experiment. 


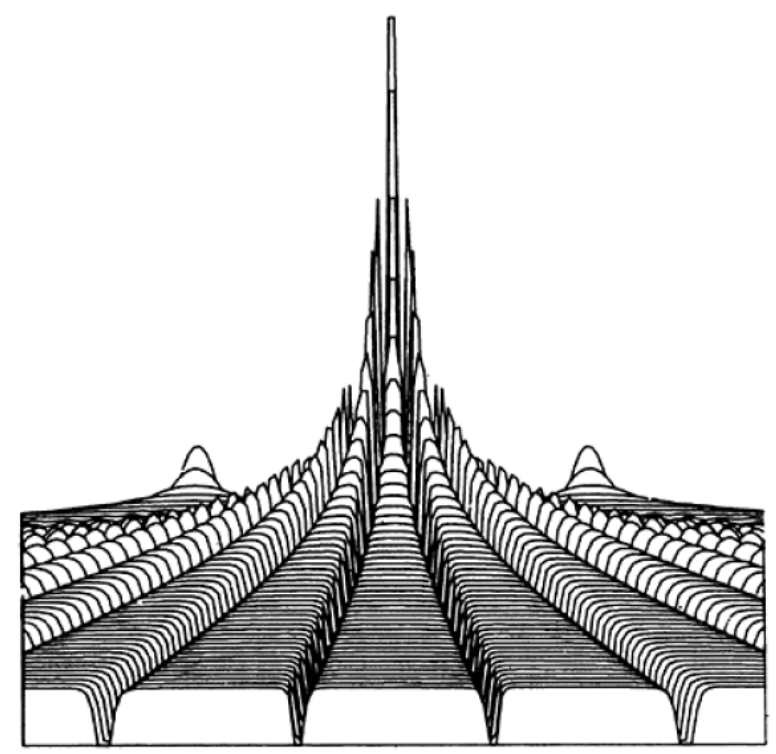

Figure 1: Quantum potential for two Gaussian slits

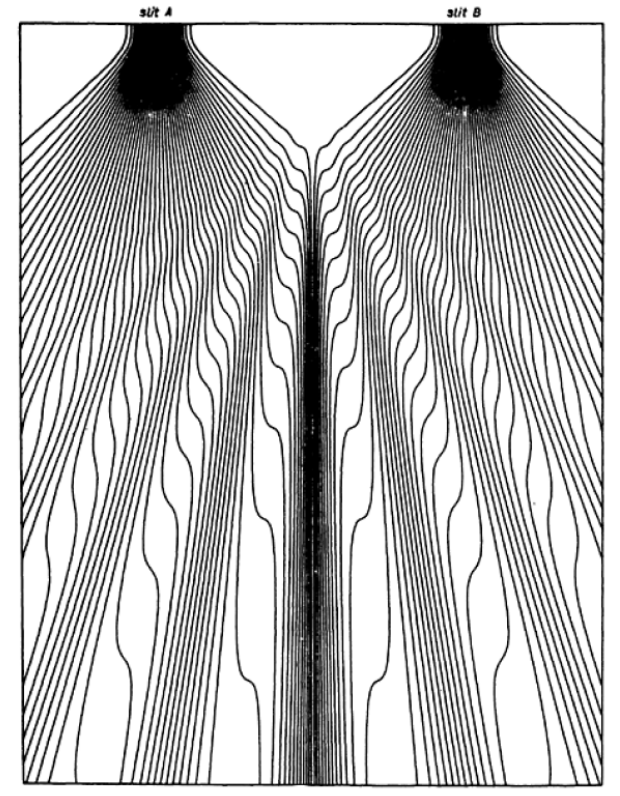

Figure 2: Trajectories for two Gaussian slits

How, then, might Bohm's theory be relevant to the mind-matter relation? It postulates that an electron is a particle, always accompanied by a new type of field, which guides its behaviour - thus the name "pilot wave theory" which is sometimes used. Jack Sarfatti has described the Bohmian electron imaginatively by saying that it consists of a "thought-like" pilot wave, guiding a "rock-like" particle. This metaphor suggests that matter at the quantum level is fundamentally different from the sort of mechanical matter of classical physics that is presupposed in philosophy of mind by 
typical materialists. If even the basic elements that constitute us have "thought-like" and "rock-like" aspects, then it is perhaps not so surprising that a very complex aggregate of such elements (such as a human being) has a body, accompanied by a mind that guides it.

But, one might think, this is merely a vague metaphor. Now, Bohm himself realized in the early 1980s that the pilot wave might be more literally "thought-like" in a very interesting sense. He considered the mathematical expression of the socalled quantum potential, which describes the way the pilot wave field affects the particle (eq. 4):

$$
Q=-\frac{\hbar^{2}}{2 m} \frac{\nabla^{2} R}{R}
$$

He realized that the quantum potential, and thus the effect of the wave upon the particle, only depends on the form or shape of the field, not on its size or amplitude $R$ (mathematically, the quantum potential depends only on the second spatial derivative of the amplitude $R$ of the field; $h$-bar is a constant and $m$ is the mass of the particle). He went on to suggest that the quantum wave field is literally putting form into, or informing the particle, rather than pushing and pulling it mechanically.

Note that we are here talking about information for the electron, not information for us - we are thus thinking about information as an objective commodity that exists out there in the world, independently of us, and guides physical processes. The form of the quantum wave reflects the form of the environment of the particle - for example the presence of slits in the two-slit experiment. Subtle differences in the environment of the particle are then reflected in its behaviour which is exactly what we observe in, say, the two-slit experiment or the AharonovBohm effect. What happens with the electron is somewhat analogous to a ship on autopilot, guided by radar waves that carry information about the environment of the ship. The radar waves are not pushing and pulling the ship, but rather in-forming the much greater energy of the ship.

Bohm generalized this into a notion of "active information" - which applies in situations where a form with small energy enters and informs a larger energy. We see this not only with various artificial devices, but also in the way the form of the DNA 
molecule informs biological processes, and even in the way forms act in human subjective experience (for example, seeing the form of a shadow in a dark night and interpreting it as "danger" may give rise to a powerful psycho-somatic reaction). Indeed, Bohm $(1989,1990)$ sketched how the active information approach could be developed into a theory of mind and matter.

He suggested that we understand mental states as involving a hierarchy of levels of active information. We not merely think about objects in the external world, but we can also become aware of our thinking. He suggested that such meta-level awareness typically involves a higher level of thought. This higher level gathers information about the lower level. But because its essential nature is active information, it not merely makes a passive representation of the lower level. Rather, the higher level also acts to organize the lower level, a bit analogously to the way the active information in the pilot wave acts to organize the movement of the particle. And of course, we can become aware of this higher level of thought from a yet higher level, and so on.

How does then mind, understood as a hierarchy of levels of active information, connect with matter in the Bohmian scheme? First of all, Bohm suggested that it is natural to extend the quantum ontology. So just as there is a pilot wave that guides the particle, there can be a super-pilot wave that guides the $1^{\text {st }}$ order pilot wave, and so on. He claimed that such an extension is "natural" from the mathematical point of view; Bohm and Hiley briefly discuss such extensions in the context of quantum field (rather than particle) theory $(1993: 378-381 ; 385)$. Now it seems that we have two hierarchies, one for mind and another for matter. Bohm's next step was to postulate that these are the same hierarchy, so that there is only one hierarchy. This then allows, at least in principle, for a new way of understanding how mind can affect the body. Information at a given level of active information in the mind can act downwards, all the way to the active information in the pilot waves of particles in, say, the synapses or neural microtubules, and this influence can then be amplified to signals in motor cortex, leading to a physical movement of the body.

The above proposal differs strongly from the usual theories in cognitive neuroscience. Most neuroscientists ignore quantum considerations, and seek the "neural correlates of consciousness" in some macroscopic neural phenomena, which can presumably be understood in terms of classical physics. Yet Bohm is proposing that mind, understood as a hierarchy of levels of active information, is implemented in 
(or perhaps identical with) a hierarchy of quantum fields. However, these fields are not separate from the macroscopic neural processes. On the contrary the role of the former is in the end to guide the latter and to gather information about them.

Of course, it is a tremendous challenge to work out an empirically testable theory along the Bohmian lines, but these ideas provide a scheme for such an endeavour. For example, Hiley and Pylkkänen (2005) discuss the prospects of applying the Bohm scheme to Beck and Eccles's quantum model of synaptic exocytosis (for an account of the latter, see Atmanspacher 2015). While this may be a small step forward, problems remain. For instance, Henry Stapp (private communication) has pointed out that the sort of interference of the mind upon the laws of quantum mechanics that the Bohmian mind-matter scheme involves can lead to problems with special relativity. This is a challenge that future research along Bohmian lines needs to face (see also Maleeh and Amani 2012).

While the possibility of non-negligible quantum effects in the brain is often dismissed as implausible, there are interesting recent advances in quantum biology (see e.g. Ball 2011). And it is already part of mainstream neuroscience that the retina acts to amplify the effects of individual photons. Also, researchers such as Roger Penrose and Stuart Hameroff have discussed in great detail how quantum effects might play a role in neural processes via quantum coherence and collapse in neural microtubules (for recent advances with this approach, see Craddock et al. 2014) Connecting the Hameroff-Penrose work with the Bohm scheme is one potentially fruitful line for future research.

Note that Bohm introduced a new category, namely information to the debate. Is information physical or mental? He suggested that it is simultaneously both physical and mental, or has these two as its aspects. This sort of view is called a double-aspect theory in philosophy of mind. The traditional worry with doubleaspect views is that it is left into a mystery what the underlying thing, which has the aspects, is. The hypothesis that information is the fundamental, underlying feature of reality can be seen as a way to alleviate this worry.

\section{Consciousness in the quantum theoretical active information scheme}

It is well known that a major problem with both the identity theory and functionalism in philosophy of mind is that they leave out conscious experience, instead of 
explaining it. How might conscious experience fit into the active information scheme? While Bohm saw nature as a dynamic process where information plays a key dynamic role, he assumed that " $99.9 \%$ " of the activity of information is not conscious (Weber 1987). Thus, for example, he thought it obvious that the particles of physics are not conscious. But how can one then approach the hard problem of consciousness in this scheme? In other words, why is there sometimes conscious experience associated with the activity of information (as seems obvious at least with humans and higher animals)? Why doesn't all the activity of information in humans proceed "in the dark", as it seems to do in physical and biological processes in general? Bohm himself did not say much about the hard problem of consciousness (he died a little before the hard problem was made the center of attention by David Chalmers in 1994). However, I think that the most natural context to explore this issue is some version of a higher order (HO) theory of consciousness. A simple possibility would be to postulate that what makes a given mental state (or level of information or mental activity) conscious is that there exists a higher level of (typically) unconscious information, which has the content that one is in the first order mental state or activity (cf. Rosenthal 1997; Gennaro 2012).

Note also that David Chalmers (1996) famously suggested that we tackle the hard problem of consciousness with a double-aspect theory of information. The idea is that information is a fundamental feature of the world, which always has both a phenomenal and a physical aspect. Now, we could take this idea to the Bohm scheme and postulate that active information, too, has phenomenal properties. This then raises the question about what we should think about the active information in the pilot wave of an electron. Does it, too, have phenomenal properties in some sense? Bohm went as far as to say that electrons have a "primitive mind-like quality", but by "mind" he was here referring to the "activity of form", rather than conscious phenomenal experience in any full sense.

I think that it is reasonable to combine Chalmers's hypothesis to active information, but we need to restrict the hypothesis. For example, we could say that certain kind of active information (for example, a holistic active information that is analogous to quantum active information) has the potentiality for phenomenal properties, but this potentiality is actualized only in suitable circumstances (for example, when a given level of active information is the intentional target of a higher level of active information). Of course, this also opens up the possibility for genuine 
artificial consciousness. If we could implement quantum-like holistic active information in an artificial system and set of up a suitable higher-order relationship of levels in the system, phenomenal properties should actualize themselves, according to this hypothesis.

One advantage is that while Chalmers' double-aspect theory suffers from epiphenomenalism, Bohm's scheme, when modified, opens up the possibility of a genuine causal efficacy of phenomenal properties on the physical domain (see Pylkkänen 2007: 244-6; Pylkkänen, forthcoming.) Also, Chalmers thinks it an interesting possibility that some sort of activity is required for experience, and that static information (e.g. information in a thermostat in a constant state) thus is not likely to have experience associated with it (1996: 298). If we say that phenomenal properties are always properties of some kind of Bohmian active information, we could do justice to the intuition that activity is required for experience.

Bohm and Hiley emphasize that active information is quite different from Shannon information. The latter provides quantitative measure of information that represents the way in which the state of a system is uncertain to $u s$, in the sense that we can only specify probabilities of various states. In contrast, active information is not essentially related to our knowledge or lack of it. At the quantum level Bohm and Hiley refer to information that is relevant to determining the movement of the electron itself, i.e. information for the electron. Information is here seen as an "objective commodity" (cf. Dretske 1981). Bateson characterized information as "a difference that makes a difference". Bohm felt this is too broad, as strictly speaking every difference makes a difference. To restrict it, he proposed that information is a difference of form that makes a difference of content.

\section{Tononi et al.'s Integrated Information Theory of consciousness}

Much attention in consciousness studies has in recent years been given to Giulio Tononi and co-workers' Integrated Information Theory (IIT) of consciousness, as it promises to offer a principled account of what experience is and what type of physical systems can have it (for recent reviews, see Tononi and Koch 2014; Oizumi, Albantakis and Tononi 2014). Tononi et al.'s strategy is to start by identifying the essential properties of consciousness and then ask what kinds of physical mechanisms could possibly account for them. This has resulted in a mathematical theory of 
consciousness that is not restricted to neural explanation. According to many, IIT takes consciousness theorizing into a new level.

Tononi et al. think it self-evident that consciousness exists and is compositionally structured, differentiated, unified and singular. These five features (which they call EXISTENCE, COMPOSITION, INFORMATION, INTEGRATION, EXCLUSION) are the phenomenological axioms of their theory. They then make a number of postulates to account for these axioms or features. Finally, they propose some identities. Let us first consider the five axioms and the postulates that account for them in turn.

EXISTENCE. To account for the existence of consciousness (from its own intrinsic perspective) they propose that there has to be a system of mechanisms with a cause-effect power upon itself (i.e. intrinsically, independent of extrinsic causes and effects).

COMPOSITION. To account for the compositional structure of consciousness (i.e. that each experience is composed of many phenomenological distinctions / has multiple aspects), it must be possible to compose elementary mechanisms into higherorder mechanisms which have irreducible causes and effects.

INFORMATION. To account for the fact that consciousness is differentiated (i.e. that each experience has a specific set of phenomenological distinctions / differs in its particular way from others), a system of mechanisms must specify a differentiated conceptual structure via a process of in-forming. Through its causeeffect power, a system of mechanisms in a state gives rise to a form or "informs" a conceptual structure in the space of possibilities. A concept is how each mechanism within the system specifies the probability of past-future states of the system (causeeffect repertoire). A conceptual structure then is the set of concepts specified by the mechanisms of the system in various combinations.

INTEGRATION. To account for the irreducible unity of consciousness (i.e. that each experience is strongly irreducible to non-interdependent components), there has to be integrated information, in the sense that the conceptual structure specified by the system is irreducible to that specified by non-interdependent sub-systems. The presence of integration (characterized by big phi or $\Phi$ ) means that a partitioning of a system of mechanisms would destroy several cause-effect repertoires and change others. 
EXCLUSION. Finally, to account for the singularity of consciousness in content and spatio-temporal grain (i.e. that there is no superposition of multiple experiences), the system of mechanisms must be such that there is no superposition of conceptual structures over elements and spatio-temporal grain.

In this framework Tononi and Koch define a complex as the system of mechanisms that generates a maximally irreducible conceptual structure or a quale. The central identity of IIT then states that a conscious experience is identical to a maximally irreducible conceptual structure: “..the quale completely specifies both its quality (the set of concepts in the quale is the content of consciousness) and its quantity (the value of irreducibility $\Phi_{\max }$ of the quale is the level of consciousness") (Tononi and Koch 2014).

\section{Integrated Information Theory of consciousness and the Bohmian mind- matter scheme: a brief comparison}

It is not a straightforward task to compare and contrast Tononi's IIT with Bohm's mind-matter scheme. For one thing, Tononi's theory is a fairly well developed mathematical theory, with a number of new concepts, making it somewhat difficult to understand. In contrast, Bohm's approach remains a scheme. There have been some developments related to it (see e.g. Smith 2003), but quite a bit more is needed before it can be seen as a full alternative to IIT. So in this preliminary attempt to compare these frameworks, I will just draw attention to some points of mutual relevance, which can then act as a basis for further development.

Both Tononi and Bohm use the concept of information in a way different than it is used in communication theory. For Tononi, information refers to how a system of mechanisms in a state, through its cause-effect power, gives rise to a form ("informs" a conceptual structure) in the space of possibilities. For Bohm active information refers to situations when a form (carrying a little energy) enters and literally in-forms a larger energy. This is an interesting similarity between the schemes, although there are subtle differences.

Both Tononi and Bohm build upon Bateson's idea of information as a "difference that makes a difference". For Tononi the key point is that to generate information, a mechanism must make a difference in the sense that it constrains the states of a system that can be its possible causes and effects. Neurons and logic gates 
made of transistors qualify as such information generators. When it comes to consciousness, Tononi notes that for conscious experience to be able to exist from its own intrinsic experience, the system of mechanisms that generates such information and experience must have cause-effect power within itself (i.e. intrinsically, independent of extrinsic causes and effects).

Bohm, too starts from Bateson's notion that information is a difference that makes a difference. But, Bohm notes, every difference makes a difference. Bateson's notion thus has to be constrained. Bohm does this by saying that information is a difference of form that makes a difference of content (Bohm 1989). To make this notion better suitable for explaining consciousness I suggest that we say that information underlying consciousness is a difference that makes a difference of (at least potentially) conscious phenomenal or intentional content.

Finally, let us consider the idea that consciousness requires integrated information (in the sense that the conceptual structure or quale specified by the system is irreducible to that specified by non-interdependent sub-systems). Now, there is an interesting sense in which Bohmian quantum theoretical active information can be understood as integrated information. We can see this by considering the Nbody system in the Bohm theory, where, first of all, the behavior of each particle may depend nonlocally on all the others, no matter how far away they may be. Nonlocality is an important new feature of the quantum theory, but Bohm used to emphasize that there is yet another feature that is even more radical. For in the Bohm theory there can be a nonlocal connection between particles that depends on the quantum state of the whole, in a way that cannot be expressed in terms of the relationships of the particles alone (see Bohm and Hiley 1987: 332). This quantum state of the whole, described by the many-body wave function, evolves in time according to the Schrödinger equation, which led Bohm and Hiley to write:

Something with this sort of independent dynamical significance that refers to the whole system and that is not reducible to a property of the parts and their inter-relationships is thus playing a key role in the theory. ... this is the most fundamental new ontological feature implied by quantum theory. (Bohm and Hiley 1987, 332) 
Recall that according to Bohm and Hiley this state of the whole has to be understood as active information. I think it is a promising hypothesis that this provides a prototype model for the sort of "integrated information" that we also encounter, in much more complex form, in conscious experience. In future research my aim is to work out a view of consciousness, where Bohmian quantum-like integrated information is a key concept characterizing the holistic features of conscious experience. With the help of some version of a higher-order theory of consciousness (e.g. Gennaro 2012) it is hoped that one is able to provide an account of what it is that makes non-conscious mental states (understood as integrated active information) conscious. (For an attempt to apply a higher order theory of consciousness to the Hameroff-Penrose scheme, see Hameroff, Gennaro and Pylkkänen 2014.)

Acknowledgments. Sections 2 and 3 of this article contain text that has been adapted from a longer article by the author (Pylkkänen, P. (forthcoming)).

\section{Literature}

Atmanspacher, H. (2015). Quantum Approaches to Consciousness, The Stanford Encyclopedia of Philosophy, E. N. Zalta (ed.), URL = $<$ http://plato.stanford.edu/archives/sum2015/entries/qt-consciousness/>.

Baars, B.J., Banks, W.B. and Newman, J.B. eds. (2003) Essential Sources in the Scientific Study of Consciousness. MIT Press, Cambridge, MA.

Bacciagaluppi, G. and Valentini, A. (2009) Quantum Theory at a Crossroads: Reconsidering the 1927 Solvay Conference. Cambridge University Press, Cambridge.

Ball, P. (2011) The dawn of quantum biology, Nature 474, 272-274.

Barwise, J. and Seligman, J. (1997) Information Flow: The Logic of Distributed Systems. Cambridge University Press, Cambridge.

Bohm, D., (1952) A Suggested Interpretation of the Quantum Theory in Terms of "Hidden Variables" I and II, Phys. Rev., 85 (2): 166-179 and 180-193.

Bohm, D. (1989) Meaning and Information. In P.Pylkkänen ed. The Search for Meaning. Crucible, Wellingborough.

Bohm, D. (1990): A new theory of the relationship of mind and matter, Philosophical Psychology 3, pp. 271-286.

Bohm, David and Hiley, Basil J. (1987) An Ontological Basis for Quantum Theory: I. Non-relativistic Particle Systems. Phys. Rep. 144 (6): 323-348. 
Bohm, D. \& Hiley, B.J. (1993): The Undivided Universe. An Ontological Interpretation of Quantum Theory. Routledge, London.

Chalmers, D. (1996) The Conscious Mind: In Search of a Fundamental Theory. Oxford University Press, Oxford.

Chalmers, D. ed. (2002) Philosophy of Mind: Classical and Contemporary Readings. Oxford University Press, Oxford.

Craddock T.J.A., Friesen D., Mane J., Hameroff S., Tuszynski J.A. (2014) The feasibility of coherent energy transfer in microtubules. J. R. Soc. Interface 11: 20140677. http://dx.doi.org/10.1098/rsif.2014.0677

Dretske, F. (1981) Knowledge and the Flow of Information. MIT Press, Cambridge, MA.

Dürr, D., Goldstein, S. and Zanghi, N. (2013) Quantum Physics Without Quantum Philosophy. Springer, Berlin.

Gennaro, R. (2012) The Consciousness Paradox. MIT Press, Cambridge, MA

Goldstein, Sheldon, (2013) Bohmian Mechanics, The Stanford Encyclopedia of Philosophy, Edward N. Zalta (ed.), URL = http://plato.stanford.edu/archives/spr2013/entries/qm-bohm/

Hiley, B.J.; Pylkkänen, P. (2005) Can mind affect matter via active information? Mind and Matter, 3, 2, 7-26. URL = http://www.mindmatter.de/resources/pdf/hileywww.pdf

Hameroff, S., Gennaro, R. and Pylkkänen, P., (2014) HOT to DOT: A 'Deeper-Order Thought' Theory of Consciousness. In Brain, Mind and Cosmos [e-book], Trident.

Holland, P. (2011) A quantum of history. Contemp. Phys. 52, 355.

Lycan, W.G. and Prinz, J.J. (2008) Mind and Cognition: An Anthology. $3^{\text {rd }}$ Edition. Blackwell, Oxford.

Maleeh, R. and Amani, P., (2012) Bohm's theory of the relationship of mind and matter revisited. Neuroquantology 10, 150-163.

Ney, A. and Albert, D. eds. (2013) The Wave Function: Essays on the Metaphysics of Quantum Mechanics. Oxford University Press, Oxford.

Oizumi, M., L. Albantakis and G. Tononi (2014). "From the phenomenology to the mechanisms of consciousness: integrated information theory 3.0." PLoS Comput Biol 10 (5): e1003588.

Penrose, R. (1989) The Emperor's New Mind. Oxford University Press, Oxford.

Pylkkänen, P. (2007) Mind, Matter and the Implicate Order. Springer Frontiers 
Collection, Berlin and New York.

Pylkkänen, P. (forthcoming) Is there room in quantum ontology for a genuine causal role of consciousness? Forthcoming in A. Khrennikov \& Emmanuel Haven eds., The Palgrave Handbook of Quantum Models in Social Science. Palgrave Macmillan.

Rosenthal, D. M., (1997) A theory of consciousness. In The Nature of Consciousness; N. Block, O. Flanagan, G. Guüzeldere, Eds.; MIT Press, Cambridge, MA..

Smith, Q., (2003) Why cognitive scientists cannot ignore quantum mechanics? In Consciousness: New Philosophical Perspectives; Smith, Q., A. Jokic Eds.; Oxford University Press: Oxford.

Tononi, G. and Koch, C (2014) Consciousness: Here, There but not Everywhere. arXiv:1405.7089v1 [q-bio.NC]

Wang, Z., Busemeyer, J. R., Atmanspacher, H., \& Pothos, E. M. (2013) The potential of using quantum theory to build models of cognition. Topics in Cognitive Science, 5, $672-688$.

Weber, R. (1987) Meaning as being in the implicate order philosophy of David Bohm: a conversation, in B.J. Hiley and F.D. Peat Eds. Quantum Implications: Essays in honour of David Bohm. London, Routledge,. 